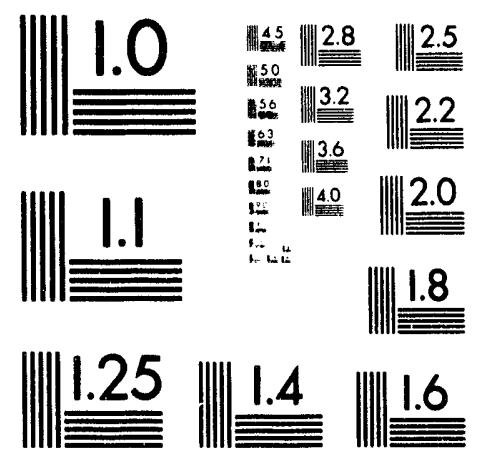



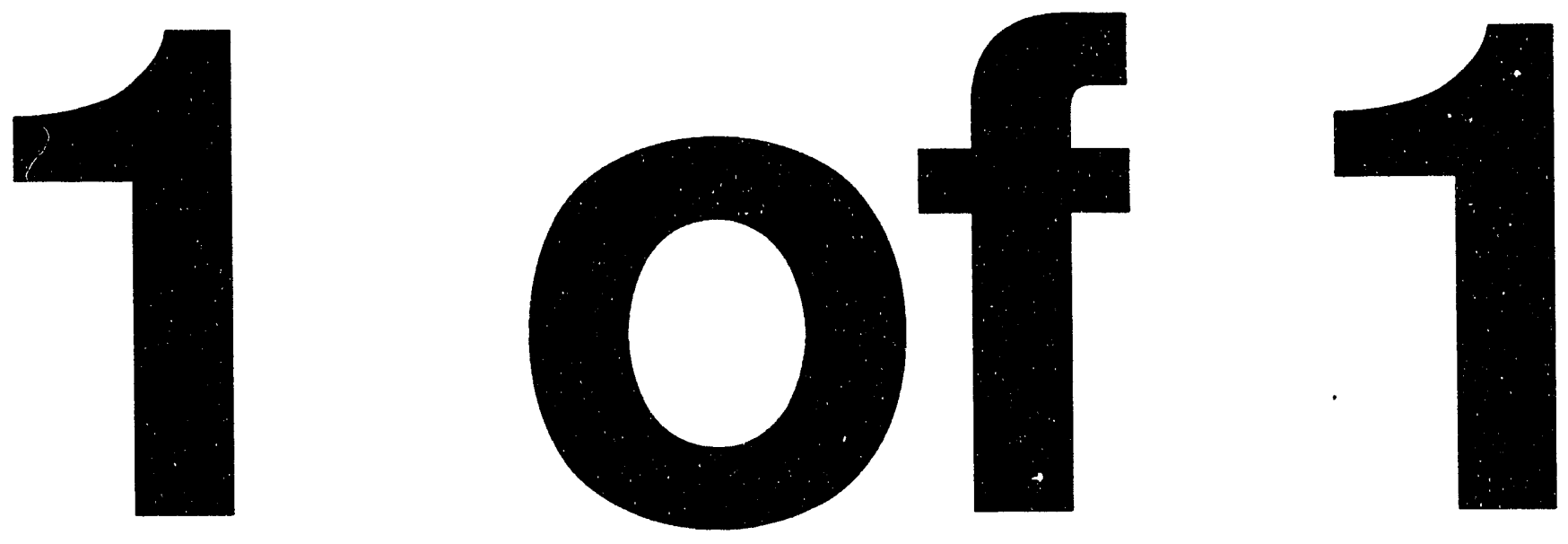


\section{INSTITUTE FOR FUSION STUDIES}<smiles>[14CH3][14CH3]</smiles>

DOE/ET-53088-616

IFSR \#616

Finite Beta Plasma Equilibrium in Toroidally Linked Mirrors

V.I. Ilgisonis, ${ }^{a)}$ H.L. Berk, and V.P. Pastukhov ${ }^{a}$

Institute for Fusion Studies

The University of Texas at Austin

Austin, Texâs 78712

July 1993

4) Pcrnanent address: Institute of Nuclear Fusion, Russian Rescarch Centre

"Kurchatov institute," Moscow, 123182 Russia

\section{THE UNIVERSITY OF TEXAS}

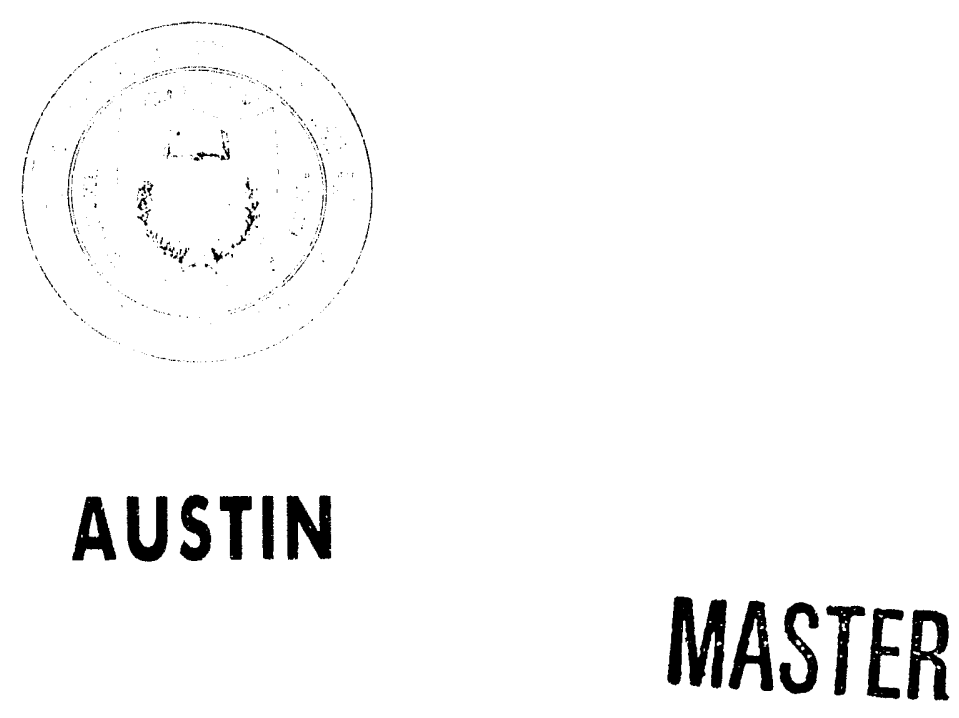

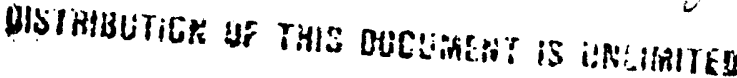




\title{
Finite Beta Plasma Equilibrium in Toroidally Linked Mirrors
}

\author{
V.I. Ilgisonis, $\left.{ }^{a}\right)$ H.L. Berk, and V.P. Pastukhov ${ }^{a)}$ \\ Institute for Fusion Studies \\ The University of Texas at Austin \\ Austin, Texas 78712
}

\begin{abstract}
The problem of finite pressure plasma equilibrium in a system with closed mag. netic field lines consisting of quadrupole mirrors linked by simple toroidal cells with elliptical cross-sections is analyzed. An appropriate analytical procedure is developed, that uses conformal mapping techniques, which enables one to obtain the magnetic field structure for the free boundary equilibrium problem. This method has general applicability for finding analytic solutions of the two-dimensional Dirichlet problem outside of an arbitrary closed contour. Using this method, the deformations of the plasma equilibrium configuration due to finite plasma pressure in the toroidal cell are calculated analytically to the second order in $\lambda$-expansion, where $\lambda \sim \beta / \epsilon E, \beta$ is the ratio of plasma pressure to the magnetic field pressure, $\epsilon$ is the inverse aspect ratio and $E$ is the ellipticity of the plasma cross-section. The outer displacement of the plasma column is shown to depend nonlinearly on the increase of plasma pressure, and does not prevent the achievement of substantial $\beta \sim 10 \%$ in the toroidal cells.

PACS $52.5 .5 \mathrm{HC}$
\end{abstract}

\footnotetext{
"Permanent address: Institute of Nuclear Fusion, Russian Research Centre "Kurchatov Institute" Moscow 123182, Russia
} 


\section{Introduction}

Toroidal linkage is expected to decrease the particle loss level from mirrors and increase the electron temperature of confined plasma. ${ }^{1,2}$ These features are the basis of the neutron source project recently suggested. ${ }^{3}$ In this last reference the neutron source is composed of two parallel pairs of minimum- $B$ mirrors linked by simple toroidal cells. The special features of this design are the enhanced plasma pressure in mirrors with respect to toroidal cells, and the high ellipticity $(E \geq 10)$ of the magnetic flux tube cross-sections in the toroidal cells. This high ellipticity is a favorable feature ${ }^{3}$ which enhances the permissible toroidal $\beta$ (the ratio of plasma pressure to magnetic field pressure), and reduces neoclassical transport. Compared tw open-ended systems, the closed field line feature leads to an increase of neutron source efficiency in 10-20 times. Further, like mirror systems, operation at high $\beta \sim(1 / 2-1)$ in the mirror regions is possible. ${ }^{4,5}$ It is important to emphasize that the value of $\beta$ in toroidal cell is a crucial quantity to provide attractive parameters of a neutron source such as achieving a desired neutron fluence in the shortest time.

The presence of finite pressure plasma in the toroidal cells can result in the appearance of the additional displacement (nonuniform along the torus axis) of the plasma column. The value of such a displacement was estimated in Ref. 3 in linear- $\beta$ approximation under an assumption of very high ellipticity $E \gg 1$. However, to be sure that the estimates ${ }^{3}$ can really be used, more accurate calculations of the equilibrium plasma configuration are needed. The additional question to be solved is the character of the flux surface deformation due to multipole currents. Generally speaking, in the case of finite- $\beta$ equilibrium, such a deformation can be more important than the simple displacement of the plasma column, and hence, be a more crucial factor limiting maximum plasma pressure in toroidal cells.

In this work we presen "ather accurate calculations of the finite pressure plasma equi- 
librium configuration for the Linked Mirror Neutron Source device (LMNS) suggested in Ref. 3. All the magnetic fields and constant pressure surfaces are calculated analytically up to second order in a $\beta$-expansion for arbitrary values of ellipticity. It is shown that the nonlinear deformation of the flux surfaces due to finite plasma pressure reduces the strong influence of the $\beta$-increase on the convex shape of the plasma column within toroidal cells. To obtain the correct analytical solution for the magnetic fields, a relatively simple method of how to solve the Dirichlet's problem on the outside of an arbitrary closed ordinary curve in the cross-sectional plane, is also developed. This method is briefly described in Appendix A. The equilibrium configuration obtained can be a basis for subsequent stability analysis.

\section{Linked Mirror Geometry}

The schematic view of the Linked Mirror device magnetic configuration is presented in Figure 1. Each mirror cell, closed between cross-sections 11-1-3 and 5-7-9, looks like a pair of quadrupole mirrors such as in $2 \mathrm{XII}-\mathrm{B}^{6}$ with high pressure sustained, for example, by neutral beam injection. The magnetic field line linkage is provided by means of simple toroidal cells (cross-sections 3-4-5 and 9-10-11).

In accordance with Ref. 3 we shall begin our considerations from a vacuum magnetic configuration. which can be described in toroidal cell by the following magnetic field components:

$$
B_{X}=B_{Y}=0 ; \quad B_{S}=B_{0} ; \quad B=B_{0} /\left(1+k_{T} X\right) .
$$

We have introduced here the coordinates $\{X, Y, S\}$ in the toroidal cell, where $S$ is a longitudinal coordinate along the toroidal axis with curvature $k_{T}$, and $X$ and $Y$ are cartesian coordinates in the plane perpendicular to that axis. The corresponding metric tensor $g_{i j}$ has the components: $g_{1 j}=0 \quad(i \neq j) ; g_{11}=g_{22}=1 ; g_{33}=\left(1+k_{T} X\right)^{2}$. Below we shall use the dimensionless coordinates: $X / a \rightarrow x ; Y / b \rightarrow y ; k_{T} S \rightarrow \zeta$ where $a$ and $b$ are the minor and 
major axes of the boundary magnetic flux tube with the ellipticity $E=b / a \geq 1$. Instead of dimensional curvature $k_{T}$ we introduce the dimensionless parameter $\epsilon=k_{T} a$. We assume the parabolic distribution of initial plasma pressure $p$ in toroidal cells:

$$
p=p_{0}(1-\Psi)
$$

where $p_{0}$ is the pressure on the torus axis $x=y=0$, and

$$
\Psi \approx x^{2}+y^{2}
$$

is the label of the flux tube surface which is constant along the magnetic field line. The ellipse $\Psi=1$ corresponds to external boundary of the plasma column. For the above pressure distribution the parameter $\beta$ is defined as:

$$
\beta=\frac{2}{B_{0}^{2}}\left|\frac{d p}{d \Psi}\right|=\frac{2 p_{0}}{B_{0}^{2}} .
$$

\section{Description of the Method}

A plasma equilibrium in the closed magnetic system can be achieved under the following condition:

$$
\oint(\mathbf{B} \cdot \boldsymbol{\nabla}) \mathbf{B} \times \mathbf{B} \cdot \boldsymbol{\nabla}\left(p_{\|}+p_{\perp}\right) \frac{d \ell}{B^{5}}=0 .
$$

The subscripts " $\|$ " and " $\perp$ " correspond to the magnetic field direction, and the integration is carried out along any closed magnetic field line (in particular, along the axis of the system). Without rotational transform, condition (3) can be satisfied by means of an appropriate choice of the curvature and magnetic field magnitude along the axis. Although the geometry of the toroidal cell is prescribed as a simple toroidal field (see Sec. II), it is easy to show that the global equilibrium condition (3) can always be satisfied by an appropriate choice of plasma parameters in the mirror regions. In particular, to leading order in the paraxial expansion, condition (3) is satisfied by a specified shift of the plasma column with respect to 
the geometric axis in mirror cell, or(and) by bending the mirror cell axis (it corresponds to the closing of dipole currents). ${ }^{3}$ To the next order in the expansion (the closing of quadrupole currents) condition (3) can be satisfied by a slight elliptical deformation of constant pressure surface in the mirror midplane, and so on. Therefore, below we shall assume that the condition of global equilibrium given by Eq. (3) is always satisfied, and consider in more detail the equilibrium plasma configuration in the toroidal cells. As boundary conditions for this consideration we shall fix the form and position of constant pressure surfaces in cross-sections 3,5,9, and 11 (see Fig. 1) between toroidal cells and mirrors. Although this is only approximate, and the increase of the plasma pressure in the toroidal cells is able to change the plasma configuration in the mirrors as well, such a change is weak due to higher plasma pressure in the mirrors with respect to toroidal cells.

Let us roughly estimate the effect of the presence of a finite- $\beta$ plasma in toroidal cell. To provide the needed equilibrium the perpendicular current

$$
\mathbf{j}_{\perp}=\frac{[\mathbf{B} \times \nabla p]}{B^{2}} \sim \beta \frac{B_{0}}{a}
$$

is generated together with corresponding Pfirsch-Schlüter current

$$
\mathbf{j}_{\|} \sim \beta \frac{B_{0}}{a E} .
$$

Such an estimate can be made because the main part of the gradient of the magnitude of the magnetic field is in the $x$-direction. This toroidal current generates the additional poloidal magnetic field $B_{\perp} \sim 3 B_{0} / E$, and in its turn the perpendicular current generates an addition $\delta B_{S} \sim \beta B_{0}$ to the toroidal field.

Although the above currents appear due to toroidal curvature, the toroidicity parameter $\epsilon$ does not appear immediately (the curvature comes here in the combination $\zeta=k_{T} S$ only). It means that the $\beta$-deformations of the field structure (and hence, of flux surfaces) appear earlier than toroidal corrections, and are the principal ones. Therefore, it is reasonable to analyze $\beta$-corrections to lowest order in the $\epsilon$-expansion. 
The first 3 -correction to $\Psi$ should be found from the equation

$$
\mathbf{B} \cdot \boldsymbol{\nabla} \Psi=0
$$

Seeking $\Psi$ as the sum $\Psi \approx \Psi_{0}+\Psi_{1}$ where $\Psi=x^{2}+y^{2}\left|\Psi_{1}\right| \leq\left|\Psi_{0}\right|$, we find from our estimates of $\mathbf{B}_{\perp}$ and Eq. (4) that

$$
\epsilon \partial_{\zeta} \Psi_{1} \sim \frac{\beta}{E} \partial_{x, y} \Psi_{0}
$$

Hence. the magnitude of $\Psi_{1}$ appears to be of order $\sim \lambda \Psi_{0}$, where $\lambda \approx \beta / \epsilon E$. From this it follows that $\lambda$ is the real expansion parameter rather than $\beta$. Hence, we should seek $\Psi$ as an expansion series in powers of $\lambda$. To find the second order correction to $\Psi$ it is necessary to repeat the procedure of solving the equilibrium equation, i.e. to find the next order corrections to currents, magnetic field components, and so on.

The value of $\lambda$ can be estimated from the simple topological consideration. Indeed, to provide the L.MNS geometry drawn in Fig. 1 it is necessary to satisfy to the inequality

$$
b+\Delta, \leq \frac{1}{k_{T}}
$$

Where $\lrcorner_{s}$ is the thickness of magnetic coils and shield. Condition (5) guarantees the presence of some gap between cross-sections 1 and 7 of L.MNS. Supposing $\Delta_{s} \approx(0.5-1.0) b$, we find casily from $(5) \epsilon E \leq(0.5-0.7)<1$, and hence $\lambda \geq(1.5-2.0) \beta>\beta$. In addition to $E$ it is convenient to introduce $\tilde{E}=E+1$ (such a quantity appears in the detailed calculations) and to determine $\lambda$ as

$$
\lambda=\frac{\beta}{\epsilon \widetilde{E}} .
$$

Below we shall convert from a set of parameters $\{\epsilon, \beta, E\}$ to another set $\{\epsilon, \lambda, \tilde{E}\}$, assuming the following ordering

$$
\epsilon \ll \lambda \leq 1
$$

for arbitrary $\tilde{E} \geq 2 \quad(E \geq 1)$. 


\section{General Formalism}

In accordance with the consideration given in the above section, we shall consider the plasma equilibrium configuration parameters as series in $\lambda$ :

$$
\begin{gathered}
\Psi=\sum_{n=0}^{\infty} \lambda^{n} \Psi_{n}(x, y, \zeta)+\epsilon \ldots, \\
B_{X}=\frac{\beta B_{0}}{\widetilde{E}}\left(\sum_{n=0}^{\infty} \lambda^{n} \chi_{n}(x, y, \zeta)+\epsilon \ldots\right), \\
B_{Y}=\frac{\beta B_{0}}{\widetilde{E}}\left(\sum_{n=0}^{\infty} \lambda^{n} \Upsilon_{n}(x, y, \zeta)+\epsilon \ldots\right), \\
B_{S}=B_{0}\left(1+\frac{1}{2} \beta \sum_{n=0}^{\infty} \lambda^{n} Z_{n}(x, y, \zeta)+\epsilon^{2} \ldots\right) .
\end{gathered}
$$

Neglected terms are denoted here by dots.

The components $\Psi_{n}$ of the function $\Psi$ should be found from Eq. (4) which, to lowest order in $\epsilon$, takes the form:

$$
\frac{B_{0} \epsilon}{a}\left\{\Psi_{0}^{\prime}+\sum_{n=1}^{\infty} \lambda^{n}\left(\Psi_{n}^{\prime}+\sum_{m=0}^{n-1}\left(\chi_{m} \partial_{x}+\frac{\Upsilon_{m}}{E} \partial_{y}\right) \Psi_{n-m-1}\right)\right\}=0 .
$$

Here and below the prime denotes the partial derivative with respect to $\zeta$.

To solve this equation up to the certain order $n$, the function $\Psi_{0}$ must be given, and components $\gamma_{m}, \Upsilon_{m}$ where $m=0,1, \ldots,(n-1)$, must be calculated. The currents determining the magnetic field should be found from the equilibrium equations:

$$
\begin{aligned}
& \mathbf{j}_{\perp}=\frac{\mathbf{B} \times \nabla p}{B^{2}}, \\
& \mathbf{B} \cdot \nabla \frac{\mathbf{j}_{\|}}{B}=\frac{2 \nabla B \times \mathbf{B}}{B^{3}} \cdot \nabla p .
\end{aligned}
$$

Expanding the parallel current in a series

$$
\mathbf{j}_{\|}=\frac{\beta B_{0}}{a} \sum_{n=0}^{\infty} \lambda^{n} J_{n}(x, y, \zeta),
$$


we can rewrite (9) in the following form:

$$
\begin{aligned}
J_{0}^{\prime}+\frac{1}{E} \partial_{y} \Psi_{0}+\sum_{n=1}^{\infty} \lambda^{n}\left\{J_{n}^{\prime}+\frac{1}{E} \partial_{y} \Psi_{n}\right. \\
\left.+\sum_{m=0}^{n-1}\left(\left(\chi_{m} \partial_{x}+\frac{\Upsilon_{m}}{E} \partial_{y}\right) J_{n-m-1}+\frac{\tilde{E}}{2 E}\left[Z_{m}, \Psi_{n-m-1}\right]\right)\right\}=0 .
\end{aligned}
$$

The Poisson's brackets for scalars $S, Q$ are defined as

$$
[S, Q]=\partial_{y} S \partial_{x} Q-\partial_{x} S \partial_{y} Q
$$

The magnetic field components should be determined from Maxwell's equations

$$
\operatorname{rot} \mathbf{B}=\mathbf{j} ; \quad \operatorname{div} \mathbf{B}=0
$$

To leading order in $\epsilon$ the last equation (11) can always be satisfied by specifying the poloidal field through a single $S$-component of vector potential $A_{S}$, that gives

$$
B_{X} \approx \frac{1}{a E} \partial_{y} A_{S} ; \quad B_{Y} \approx-\frac{1}{a} \partial_{x} A_{S}
$$

If we take

$$
A_{S}=a \beta B_{0} \frac{E}{\tilde{E}} \sum_{n=0}^{\infty} \lambda^{n} A_{n}
$$

we find

$$
\chi_{n}=\frac{\partial A_{n}}{\partial y}, \Upsilon_{n}=-\frac{\partial A_{n}}{\partial x} E
$$

and then we can express

$$
\mathbf{j}_{\|} \approx a^{-1}\left(\partial_{x} B_{Y}-E^{-1} \partial_{y} B_{X}\right)
$$

through $A_{S}$ as well. This procedure leads to the Poisson's equation

$$
\sum_{n=0}^{\infty} \lambda^{n}\left(J_{n}+\frac{E}{\tilde{E}} \Delta_{\perp} A_{n}\right)=0,
$$

where $\lrcorner_{\perp}=\partial^{2} / \partial x^{2}+E^{-2} \partial^{2} / \partial y^{2}$ is dimensionless $2 D$ Laplacian operator. 
In accordance with Eq. (8), the perpendicular current determines the toroidal field:

$$
\begin{aligned}
\frac{1}{E} \partial_{y} B_{S} & =\frac{\beta B_{0}}{2} \sum_{n=0}^{\infty} \frac{\lambda^{n}}{E} \partial_{y} \Psi_{n}-\epsilon \beta B_{0} \frac{E}{\tilde{E}} \sum_{n=0}^{\infty} \lambda^{n} \partial_{x} A_{n}^{\prime}, \\
\partial_{x} B_{S} & =\frac{\beta B_{0}}{2} \sum_{n=0}^{\infty} \lambda^{n} \partial_{x} \Psi_{n}+\epsilon \frac{\beta B_{0}}{\tilde{E}} \sum_{n=0}^{\infty} \lambda^{n} \partial_{y} A_{n}^{\prime} .
\end{aligned}
$$

The last item on the right side of both these equations is always the quantity of order $\epsilon$ with respect to first one, therefore it should be omitted here. Then the systems of equations defined by (13) can be easily integrated:

$$
B_{S}=\frac{1}{2} \beta B_{0} \sum_{n=0}^{\infty} \lambda^{n} \Psi_{n}+F(\zeta) .
$$

The arbitrariness of a function $F$ is removed by the boundary condition. Taking into account the absence of induced field at infinity and that asymptotically $B_{S} \rightarrow B_{0}$ as $\lambda \rightarrow 0$, we find from Eqs. (6) and (14) that $Z_{n}=\Psi_{n}$ and, hence,

$$
B_{\mathcal{S}}=B_{0}\left(1+\frac{3}{2}(\Psi-1)+\epsilon^{2} \ldots\right) .
$$

Thus we have determined one set of variables $\left(Z_{n}\right)$. In addition, using (12) we can also climinate the current components $J_{n}$ from Eq. (10). Then the set of equilibrium equations (4). (8). (9). and (11) results in two independent scalar equations:

$$
\begin{gathered}
\Psi_{0}^{\prime}+\sum_{n=1}^{\infty} \lambda^{n}\left(\Psi_{n}^{\prime}+\sum_{m=0}^{n-1}\left[A_{m}, \Psi_{n-m-1}\right]\right)=0 \\
\frac{1}{E} \partial_{y} \Psi_{0}-\frac{E}{\tilde{E}} \Delta_{\perp} A_{0}^{\prime}+\sum_{n=1}^{\infty} \lambda^{n}\left(\frac{1}{E} \partial_{y} \Psi_{n}-\frac{E}{\tilde{E}} \Delta_{\perp} A_{n}^{\prime}\right. \\
\left.+\sum_{m=0}^{n-1}\left(\frac{E}{\tilde{E}}\left[\Delta_{\perp} A_{n-m-1}, A_{m}\right]+\frac{\tilde{E}}{2 E}\left[\Psi_{m}, \Psi_{n-m-1}\right]\right)\right)=0 .
\end{gathered}
$$

To these equations it is necessary to specify boundary conditions. In regards to the longit11dinal dependence (on $\zeta$ ), we may use the above assumption that $\Psi(x, y)$ is given at the ends of the toroidal cell, that is

$$
\Psi_{n}(\zeta=0, \pi)=0 \quad(\forall n>0)
$$




$$
A_{n}(\zeta=\pi / 2)=0 \quad(\forall n \geq 0)
$$

The second condition (18) was a consequence of the symmetry of the system being considered.

As regards the dependence on perpendicular coordinates $(x, y)$ let us note that Eqs. (16) and (17) describe the equilibrium configuration inside the plasma, therefore, Eq. (17) is valid up to the external plasma boundary $\Psi=1$. Outside the plasma there is no reason to introduce the $\Psi$-function, and instead of Eq. (17) we should use the equation

$$
\Delta_{\perp} A_{n}=0
$$

The solution of the vac $1 \mathrm{~m} \mathrm{Eq.} \mathrm{(19)} \mathrm{must} \mathrm{be} \mathrm{matche.} \mathrm{o} \mathrm{the} \mathrm{solution} \mathrm{of} \mathrm{internal} \mathrm{region} \mathrm{given}$ by Eqs. (16) and (17) at the boundary surface $\Psi=1$ together with partial derivatives of $A_{n}$ $(\forall n \geq 0)$ due to the continuity of the magnetic field components. In addition we must take into account the absence of an induced magnetic field at infinity. Finally, these conditions can be formalized as

$$
\forall n \geq\left. 0 \quad \partial_{x, y} A_{n}\right|_{\Psi=1-0} ^{\Psi=1+0}=0 ;\left.\quad \partial_{x, y} A_{n}\right|_{x^{2}+y^{2}-\infty} \rightarrow 0
$$

Equations (16). (17), and (19), together with boundary conditions (18) and (20), entirely determine the plasma equilibrium problem being considered.

\section{Iterative Solution}

We shall find the solution of Eqs. (16) and (17) to different orders of $\lambda$.

\section{A. First order}

Starting from the given form (2) of magnetic surface, we obtain to the leading order of Eq. (17)

$$
\lrcorner_{\perp} A_{0}^{\prime}=2 y \frac{\tilde{E}}{E^{2}}
$$


or, integrating over $\zeta$ with condition (18),

$$
\Delta_{\perp} A_{0}=2 y \frac{\tilde{E}}{E^{2}}\left(\zeta-\frac{\pi}{2}\right) .
$$

The internal solution for $A_{0}$ can be found as a power series, and due to symmetry of LMNS configuration with respect to the horizontal plane $y=0$, we need only keep the terms with odd powers of $y$,

$$
A_{0}=a_{01} y+a_{11} x y+a_{21} x^{2} y+a_{03} y^{3}
$$

The quantities $a_{i}$, in (22) do not depend on perpendicular coordinates $x, y$. By satisfying Eq. (21) we find the relation

$$
a_{21} E^{2}+3 a_{03}=\tilde{E}(\zeta-\pi / 2)
$$

The other coefficients must be determined by matching to the external solution.

It is more convenient to carry out this matching directly for field components than for their potential. berause they are continuous at the boundary $\Psi=1$, and must disappear at the infinity (20). Then, instead of (22) we can write

$$
\begin{aligned}
& \chi_{0}=a_{01}+a_{11} x+a_{21} x^{2}+3 a_{03} y^{2}, \\
& \Upsilon_{0}=-E a_{11} y-2 E a_{21} x y
\end{aligned}
$$

inside the plasma. Outside the plasma $\chi_{0}, \Upsilon_{0}$, as well as $A_{0}$, are also harmonic functions, but due to quite different behavior of internal and external solutions, it is usually a difficult problem to carry out analytically the needed connection except for simple boundaries (e.g. a circle) or for particular solutions (see, e.g., Ref. 7). Of course, there is no such problem in a case when the internal solution must vanish at the boundary ${ }^{8}$ and hence, the exterior field analysis can be omitted. 9.10

In Appendix $t$ we present a method of how to obtain an external solution which can be connected to the internal for an arbitrarily specified boundary curve. It is shown that the 
coordinate transform $(x, y) \rightarrow(u, v)$ separating variables both in Laplacian operator and in the boundary condition, can always be found by means of implicit Fourier expansion. This allows us to write the external solution in the universal form

$$
\begin{aligned}
& \chi_{0}=\sum_{m=1}^{N} C_{m} e^{-m u} \cos m v, \\
& \Upsilon_{0}=\sum_{m=1}^{N} C_{m} e^{-m u} \sin m v,
\end{aligned}
$$

where constants $C_{m}$ and the number of modes $N$ in (25) are determined to provide the matching with internal solution (24) at the plasma boundary, which is expressed in terms of a polynomial of finite order.

Knowing the transform $(x, y) \rightarrow(u, v)$, we can then simply equate the internal and external solutions expressed through variables $(u, v)$ at the boundary. Two examples of how to find this transform for a given form of the boundary contour are presented in Appendix B. In particular, for an elliptical boundary with ellipticity $E$ we have $x=\cos v, y=\sin v$ at $\Psi=1$ (see the first example in Appendix B). Substituting it into (24) and using (25), we can find from (20) and (23) all the coefficients $a_{i j}$ :

$$
\begin{gathered}
a_{11}=0 ; \quad a_{01}=\pi / 2-\zeta ; \\
a_{21}=\frac{(\zeta-\pi / 2)}{\tilde{E}} ; \quad a_{03}=(\zeta-\pi / 2) \frac{1+2 E}{3 \tilde{E}} .
\end{gathered}
$$

It is easy to see that to provide the matching, it is sufficient to keep only the first two terms in the series (25). Thus, the quantity $A_{0}$ is determined entirely:

$$
A_{0}=\left(\zeta-\frac{\pi}{2}\right) y\left(-1+\frac{2}{3} y^{2}+\frac{3 x^{2}-y^{2}}{3 \tilde{E}}\right) \text {. }
$$

With $A_{0}$ known, we are able to find the first order correction to the flux function $\Psi$ from (10) and (18), that is

$$
\Psi_{1}=\zeta(\zeta-\pi) x\left(1-2 y^{2}+\frac{3 y^{2}-x^{2}}{\tilde{E}}\right) .
$$


Let us analyze the form of the boundary flux surface $\Psi(x, y)=1$ taking into account the first order correction (27). The maximum deformation of vacuum surface appears at $\zeta=\pi / 2$ (cross-sections 4 and 10 in Fig. 1), where that surface obeys the equation:

$$
x^{2}-\lambda \frac{\pi^{2}}{4} x+\lambda \frac{\pi^{2}}{4 \tilde{E}} x^{3}+y^{2}\left(1+\lambda \frac{\pi^{2}}{4} x\left(2-\frac{3}{\tilde{E}}\right)\right)=1 .
$$

The typical form of the curve (28) at the moderate value of $\lambda$ is presented in Fig. 2b. We shall consider the equilibria where plasma boundary cannot displace along $x$-axis more than the plasma minor radius. This limits the quantity $\lambda$ :

$$
\lambda \leq \lambda_{\max }=\frac{6 \tilde{E}}{\pi^{2}(\tilde{E}-4)}
$$

The main difference between the formula (29) and the estimate given in Ref. 3 is in the accurate calculation of induced poloidal magnetic field which decreases going away from the plasma center. However, for an ellipticity $\tilde{E}=13$ which was chosen in the LMNS proposal, ${ }^{3}$ the estimate $(29)$ gives a value of permissible beta just enhanced by a factor $(\tilde{E} / E)^{2} \approx 1.17$. More important is the fact that in accordance with (29) at lower $\tilde{E} \leq 4$ the plasma column cannot displace by such a large distance by any $\lambda$ (the $x$ coordinate of boundary curve (28) cannot exceed $\sqrt{\tilde{E}}$ ). Furthermore, even for $\tilde{E}>4$ the factor in front of $y^{2}$ in (28) appears to be negative at a $\lambda$ value just lower than the one given by (29). It infers a hyperbolic character for the plasma boundary instead of an elliptical one. The separatrix between elliptical and hyperbolic flux surfaces appears at $\lambda \sim \lambda_{\text {sep }}$ :

$$
\lambda_{\text {sep }}=\frac{4 \sqrt{2}}{\pi^{2}} \frac{\tilde{E}}{2 \tilde{E}-3} \sqrt{\frac{\tilde{E}-2}{\tilde{E}-3}} .
$$

For the considered structure of the magnetic field the appearance of such a separatrix is an artifact which indicates that for $\lambda \geq \lambda_{\text {sep }}$ we are beyond the applicability region of Eq. (28), and higher-order terms neglected during our $\lambda$-expansion are intrinsically important.

Since the limitation $\lambda \leq \lambda_{\text {sep }}$ is always stronger than inequality (29), it means that applicability of the plasma boundary description given by Eq. (28), is breaking down at a 
smaller value of $\lambda$ than + e value $\lambda \sim \lambda_{\max }$ given by (29). Moreover, for $\tilde{E} \leq 3.37 \mathrm{Eq}$. (30) predicts $\lambda_{\text {sep }}>1$, which indicates a basic limitation of neglecting higher-order terms in the $\lambda$ expansion.

To correct our analysis for $\lambda>\lambda_{\text {sep }}$, higher-order terms obviously need to be taken into account.

\section{B. Second order}

Substituting the first order solutions (26) and (27) into general Eq. " and integrating over $\zeta$, we find

$$
\Delta_{\perp} A_{1}=f^{\prime}\left(f\left(1-\frac{2}{3} E\right)+\frac{\pi^{2}}{3} E\right) \frac{x y}{E^{2}}
$$

where for brevity we denoted by $f$ the combination $f=\zeta(\zeta-\pi)$. To carry out the above mentioned procedures of solving Dirichlet's problem outside the plasma and the matching of internal and external solutions, we need to represent the piasma boundary deformed in accordance with (28) in a form of Fourier sum:

$$
\begin{gathered}
\Gamma: \Psi=\Psi_{0}+\lambda \Psi_{1} \approx 1: \\
\left.x\right|_{\Gamma} \approx c_{1} \quad v-\lambda f \frac{\tilde{E}-1}{\tilde{E}^{2}}\left(1+\frac{\tilde{E}-2}{2} \cos 2 v\right), \\
\left.y\right|_{\Gamma} \approx \sin v+\lambda f \frac{\tilde{E}-2}{2 \tilde{E}^{2}} \sin 2 v .
\end{gathered}
$$

It is easy to prove that Eqs. (32) are equivalent to Eq. (28) with an error of order $\lambda^{2}$. The determination of the needed coordinate transform $(x, y) \rightarrow(u, v)$ for such a boundary $\Gamma$ is similar to the second example given in Appendix B. Having found the transformation, we obtain the solution of Eq. (31) as a polynomial

$$
A_{1}=\frac{f^{\prime}}{6 \tilde{E}}\left(2 f\left(1-\frac{5}{2 \tilde{E}}\right)-\pi^{2}\left(1-\frac{1}{\tilde{E}}\right)\right) x y\left(1-y^{2}-\frac{1}{3} x^{2}+\frac{2}{3 \tilde{E}}\left(y^{2}-x^{2}\right)\right)
$$




$$
+f f^{\prime} \frac{x y}{\tilde{E}}\left(\frac{1}{\tilde{E}^{2}}\left(1+\left(1-\frac{2}{\tilde{E}}\right) x^{2}\right)+\frac{(\tilde{E}-1)(\tilde{E}-2)}{2 \tilde{E}^{2}}\left(1-2 \frac{\tilde{E}-1}{\tilde{E}} y^{2}\right)\right) .
$$

Substituting (33) into (16), we find the second order correction to the flux function in the plasma region:

$$
\begin{gathered}
\Psi_{2}=\frac{f^{2}}{4}\left\{\left(1-2 y^{2}\right)^{2}+\frac{3}{\widetilde{E}^{2}}\left(x^{2}+y^{2}\right)^{2}+\frac{4}{\tilde{E}}\left(y^{2}\left(1-2 y^{2}\right)-x^{2}\right)\right\} \\
+\frac{f}{3 \widetilde{E}}\left(f\left(1-\frac{5}{2 \tilde{E}}\right)-\pi^{2}\left(1-\frac{1}{\tilde{E}}\right)\right)\left\{y^{2}-x^{2}+2 x^{2} y^{2}+\frac{x^{4}}{3}-y^{4}+\frac{2}{3 \widetilde{E}}\left(x^{4}-6 x^{2} y^{2}+y^{4}\right)\right\} \\
-\frac{f^{2}}{\widetilde{E}^{3}}\left\{\frac{(\tilde{E}-1)(\tilde{E}-2)}{2}\left(x^{2}-y^{2}+2 y^{2} \frac{\tilde{E}-1}{\tilde{E}}\left(y^{2}-3 x^{2}\right)\right)+x^{2}-y^{2}+\left(1-\frac{2}{\widetilde{E}}\right) x^{2}\left(x^{2}-3 y^{2}\right)\right\} \cdot \text { (34) }
\end{gathered}
$$

The three terms in (34) have different physical origin. The first term is the main one, which describes the largest shift of the deformed plasma boundary (32) by the main poloidal field with potential (26). The second term arises due to additional $\left(\sim \lambda^{2}\right)$ corrections in $\mathbf{B}_{\perp}$ found from the equilibrium equation, and the third term arises by accounting for the plasma boundary deformation in the matching of the internal and external solutions. The third term arises because, to obtain the correct matching for each new order in $\lambda$, it is necessary to substitute simultaneously all the lower order solutions into the boundary condition (20).

The typical shapes of magnetic flux surfaces $\Psi \approx \Psi_{0}+\lambda \Psi_{1}+\lambda^{2} \Psi_{2}$ in the middle crosssections of the toroidal cells are presented in Figs. 2 and 3 for different values of $\lambda$ and $\tilde{E}$. Contrary to the first order approximation the invalid separatrix doesn't appear for any $\lambda \leq 1$. Therefore, in the area of applicability, all these calculations give reasonable answers. This is the first reason why we are able to restrict our calculation to second order terms in $\lambda$-expansion. The second reason is that, as can be seen from the general equations (16) and (17), the third order corrections have an additional factor $\sim 1 / \tilde{E}$, which reduces the influence of higher-order terms to only slight distortions of the flux surfaces. Thus it appears that taking into account yet higher-order terms than $\lambda^{2}$ will not change either the qualitative flux surface structure or quantitative estimates for the plasma column displacement. 
The dependence of the maximum plasma boundary displacement in the $x$-direction with increasing $\lambda$ is presented in Fig. 4. It is interesting that for reasonable values of $\tilde{E}$ such a displacement does not exceed the plasma minor radius $\left(\max _{\left.\right|_{\Gamma}}(x)<2\right.$, where $\Gamma: \Psi_{0}+\lambda \Psi_{1}+$ $\lambda^{2} \Psi_{2}=1$ ) for any $\lambda<1$; and for $\lambda \geq 1$ the expansion procedure becomes incorrect. The more favorable and significantly nonlinear dependence of the above displacement on $\lambda$ is the main difference between present results and the previous first order estimates in $\lambda$. For a concrete device in which the plasma boundary displacement could be limited by a number of physical and technical restrictions which are not obvious a-priori, the permissible value of $\lambda_{\max } \leq 1$ will be found from Fig. 4 , and the corresponding equilibrium beta is given by

$$
\beta \leq \epsilon \tilde{E} \lambda_{\max } .
$$

\section{Conclusion}

The method of solving Dirichlet's problem described in the Appendices allowed us to analytically obtain the form of the flux surface in the toroidal cell. To calculate the magnetic flux surfaces and the plasma equilibrium configurations for present day stellarators with non-circular cross-sections, advanced numerical codes are usually used. For example, the code V.MEC ${ }^{11}$ operates with 23-mode harmonic spectrum to represent the shape of plasma boundary (which looks typically like a deformed ellipse) in stellarators ATF, Heliotron-E and LHD. ${ }^{12}$ The use of the above-described variable $v$ instead of conventional poloidal angle for the Fourier expansion seems to be able to decrease the needed number of Fourier modes and to facilitate the calculational procedure due to the known behavior of the external solution. It should be emphasized that there is in principal no limitation in making such calculations to higher order in the $\lambda$-expansion; either analytically or numerically.

Let us mention that although the respective plasma boundary displacements are smaller in case of lower ellipticity (see Fig. 4), the equilibrium beta nevertheless increases with 
increase of $\tilde{E}$ (see (35)). Moreover, as can be seen from Figs. 2 and 3, the nonuniformity of different flux surface displacements appears to be more apparent in the case of low $\tilde{E}$.

We have considered the finite $\beta$ distortion of the plasma equilibrium separately in the toroidal cells. It is clear that had we analyzed the entire closed system, condition (35) could be changed because

1. the additional flux surface deformation can appear in mirror cell ${ }^{9,13}$ as well, and therefore, the form of that surfaces in cross-sections $3,5,9$, and 11 between mirrors and toroids can differ from ellipses;

2. the unfavorable flux surface distortion must be integrated along the whole system.

The first consideration will also alter the flux surface structure we have presented. However, such changes are only slight because they appear to next order of the expansion. The last of the above considerations leads effectively to modification of plasma equilibrium parameters into the mirrors and, as was mentioned in Sec. III, does not significanly result in the additional deformations of plasma configuration in the toroidal cells. For the parameters chosen in LMINS proposal ${ }^{3}\left(a \approx 2.9 \mathrm{~cm}, k_{T}=0.5 \mathrm{~m}^{-1}, \tilde{E}=13\right)$ the estimate (35) does not really limit the toroidal beta value.

Thus the presented analysis confirms the possibility of the creation of plasma configuration in the Linked Mirror device with equilibrium consideration allowing a moderate plasma pressure $(3 \leq 10 \%)$ in the toroidal cells. In the nominal design given in Ref. 3 , considerably lower toroidal beta $(\sim 2 \%)$ was taken. Hence, the equilibrium of the toroidal linkage of the proposed neutron source ${ }^{3}$ is well within equilibrium restrictions. 


\section{Appendix A: Analytical Solution of Dirichlet's Prob- lem on the Outside of an Arbitrary Given Curve}

\section{A. Problem formulation}

Let us introduce the cartesian coordinates $X, Y$ in the plane, where the closed curve $\Gamma$ is given. For definiteness we shall consider the smooth ordinary curve without self-intersections.

The Dirichlet's problem:

To find the function $\varphi(X, Y)$ on the outside of $\Gamma$ :

$$
\begin{aligned}
& \Delta \varphi=\frac{\partial^{2} \varphi}{\partial X^{2}}+\frac{\partial^{2} \varphi}{\partial Y^{2}}=0 \\
& \left.\varphi\right|_{\Gamma}=\Phi(X, Y) ;
\end{aligned}
$$

where $\Phi(X, Y)$ is the given function.

This problem can be solved by using Green's functions. However, by such a method it may be difficult to carry out the integration explicitly, and obtain the needed solution analytically. Instead. let us suppose that we have found the coordinate transform $(X, Y) \rightarrow(u, v)$, for which

1. the lines $u(X, Y)=$ const are closed curves, and $u(X, Y)=u_{0}$ describes the curve $\Gamma$;

2. Laplacian operator has the similar form

$$
\Delta \rightarrow \frac{1}{J}\left(\frac{\partial^{2}}{\partial u^{2}}+\frac{\partial^{2}}{\partial v^{2}}\right)
$$

as in cartesian coordinates;

3. Jacobian $J$ doesn't equal zero anywhere outside of $\Gamma$. 
These three requirements mean that our transform is not degenerate, and allows us to separate the variables of the Laplacian operator as well as for the boundary curve.

In this case we can immediately expand the solution of Eq. (A1) as the series

$$
\varphi(u, v)=\sum_{n=0}^{\infty}\left(\alpha_{n} e^{n u}+\beta_{n} e^{-n u}\right)\left(\mu_{n} \cos n v+\nu_{n} \sin n v\right) \text {, }
$$

where $\alpha_{n}, \beta_{n}, \mu_{n}$ and $\nu_{n}$ are constant.

Furthermore, we also represent the function $\Phi(X, Y)$ in the following series form

$$
\Phi=\sum_{n=0}^{\infty}\left(\Phi_{n} \cos n v+\tilde{\Phi}_{n} \sin n v\right)
$$

and use $(A 2)$ to determine a relationship for the coefficients:

$$
\begin{aligned}
& \mu_{n}\left(\alpha_{n} e^{n u_{0}}+\beta_{n} e^{-n u_{0}}\right)=\Phi_{n}, \\
& \nu_{n}\left(\alpha_{n} e^{n u_{0}}+\beta_{n} e^{-n u_{0}}\right)=\tilde{\Phi}_{n} .
\end{aligned}
$$

The relations (A4) don't determine all the Fourier's coefficients in expansion (A3), hence, the uniqueness of the solution (A3) requires additional boundary conditions (e.g. when the normal derivative of $\varphi$ is given at the curve $\Gamma$, or the asymptotic form of $\rho$ at the infinity is specified). At any event the initial problem (A1) and (A2) is now reduced to finding the needed coordinate transform $(X, Y) \rightarrow(u, v)$.

\section{B. Coordinate transform}

\section{Existence}

First of all we shall demonstrate that the needed transform really does exist for any curve [. We can obtain such a coordinate transform by posing a straightforward electrostatic problem. We imagine a uniform metallic cylinder with the cross-section $\Gamma$, and we put an electric charge on this cylinder to provide a finite surface charge density. Outside the cylinder an electric field appears, and its potential $\nu_{E}$ can be chosen as the effective radial 
coordinate $u$. Indeed, $\varphi_{E}(X, Y)$ is a harmonic function outside of $\Gamma,\left.\varphi_{E}\right|_{\Gamma}=$ const and all the other equipotential surfaces $\varphi_{E}=$ const are closed around the cylinder. For any such harmonic function the conjugate function $v(X, Y)$ always exists. Physically the function $v$ is to within an overall numerical factor the number of electric field lines of force on the given line $u=$ const. Such a variable $v$ is $2 \pi$-periodic and orthogonal to the variable $u$, because the electric field lines of force are orthogonal to the equipotential surfaces $u=$ const. Hence, the variables $(u, v)$ obviously satisfy requirements 1 and 2 in subsection $A$. The Jacobian of the transform $(X, Y) \rightarrow(u, v)$ cannot equal zero anywhere outside of $\Gamma$, because the electrical field is defined everywhere, and the presence of an $x$-point on the plane cross-section of the surface $\nu_{E}=$ const will be a contradiction of the maximum principle for harmonic functions, because there is no additional charge anywhere outside of $\Gamma$.

Thus, such an electrostatic analogy guarantees the presence of needed variables $(u, v)$ for any closed ordinary curve $\Gamma$. In addition, from this analogy we can find that the lines $u=$ const in any plane perpendicular to the axis of our cylinder tend to be circles at the infinity, and have the asymptotics $u \sim \ln R\left(R=\left(X^{2}+Y^{2}\right)^{1 / 2}\right)$ when $R \rightarrow \infty$.

\section{Transform construction}

Let us mention that since the lines $u=$ const are closed, the variable $v$ is angular-like. Hence the desired inverse transform can always be expressed as a Fourier series over the variable $v$ :

$$
\begin{aligned}
& X=\sum_{n=0} X_{n}(u) \cos n v, \\
& Y=\sum_{n=1} Y_{n}(u) \sin n v .
\end{aligned}
$$

For the simplicity and according to symmetry of LMNS geometry with respect to horizontal plane $Y=0$, we shall restrict here our consideration with symmetric transforms only. Therefore we have chosen the $X$ in $(5)$ as an even function of $v$, and $Y$ - as an odd one (it is 
also easy to analyze the case without symmetry). Inverting the transform (A5), we obtain:

$$
\begin{aligned}
& \frac{\partial u}{\partial X}=\frac{1}{J} \sum n Y_{n} \cos n v ; \\
& \frac{\partial v}{\partial X}=-\frac{1}{J} \sum Y_{n}^{\prime} \sin n v ; \\
& \frac{\partial u}{\partial Y}=\frac{1}{J} \sum n X_{n} \sin n v ; \\
& \frac{\partial v}{\partial Y}=\frac{1}{J} \sum X_{n}^{\prime} \cos n v ;
\end{aligned}
$$

where prime denotes the derivative with respect to $u$, and the Jacobian

$$
J=\left(\frac{\partial u}{\partial X} \frac{\partial v}{\partial Y}-\frac{\partial u}{\partial Y} \frac{\partial v}{\partial X}\right)^{-1}=\sum_{n, m=0}^{\infty}\left(n Y_{n} X_{m}{ }^{\prime} \cos n v \cos m v+n X_{n} Y_{m}{ }^{\prime} \sin n v \sin m v\right) .
$$

To provide the needed form of the Laplacian operator (see the requirement 2 in subsection A). the Cauchy-Riemann conditions must be satisfied:

$$
\frac{\partial u}{\partial X}=\frac{\partial v}{\partial Y} ; \quad \frac{\partial u}{\partial Y}=-\frac{\partial v}{\partial X} .
$$

These conditions lead immediately to the equations:

$$
\forall n \geq 0 \quad X_{n}^{\prime}=n Y_{n} ; \quad Y_{n}^{\prime}=n X_{n},
$$

and the general solution of Eqs. (A9) is

$$
\begin{aligned}
& X_{n}=C_{n} e^{n u}+\tilde{C}_{n} e^{-n u} \\
& Y_{n}=C_{n} e^{n u}-\tilde{C}_{n} e^{-n u} .
\end{aligned}
$$

The constants $C_{n}, \tilde{C}_{n}(n=0,1,2, \ldots)$ must be chosen to satisfy requirements 1 and 3 (see subsection $A)$. Using $(A 7)$ and $(A B)$ we find that the Jacobian is a non-negative function:

$$
J=\left(\frac{\partial X}{\partial v}\right)^{2}+\left(\frac{\partial Y}{\partial v}\right)^{2} \geq 0
$$


which equals zero only in case when

$$
\begin{aligned}
& \sum_{n=0} n X_{n} \sin n v=0 \\
& \sum_{n=0} n Y_{n} \cos n v=0 .
\end{aligned}
$$

Remembering the asymptotic structure of the transform of the electrostatic analogy, we find that all the coefficients

$$
C_{n>1}=0 \text {. }
$$

Only with this condition can the lines $u=$ const tend to circles at infinity, and can $u(X, Y)$ be a single-valued function. Due to (A11), if the Jacobian of such a transform is zero anywhere it will satisfy

$$
\begin{aligned}
X_{1} \sin v & =-\sum_{n=2} n X_{n} \sin n v, \\
Y_{1} \cos v & =\sum_{n=2} n X_{n} \cos n v .
\end{aligned}
$$

We will arrange that $X_{1}, Y_{1}>0$ by changing the starting point and/or the direction of the variable $v$. Since only the amplitudes $X_{1}, Y_{1}$ contain the term that increases with increase of $u$, the positivity of Jacobian is guaranteed everywhere outside of $\Gamma$, if

$$
X_{1},\left.Y_{1}\right|_{\Gamma}>\left.\sum_{n=2} n\left|X_{n}\right|\right|_{\Gamma}
$$

Otherwise, the curve $\Gamma$ can have some self-intersections. Let us note that due to (A12) being satisfied on $\Gamma$, condition (A13) has to be valid everywhere outside of $\Gamma$. Thus, all the possible singular points of our transform have to lie inside the contour $\Gamma$.

Finally, we have to find the coefficients $C_{0}, C_{1}, \tilde{C}_{n}(n \geq 1)$ by expressing the equation for the curve $\Gamma$ in terms of the Fourier harmonics of the variable $v$. Examples of how to do it are presented in Appendix B. 


\section{Appendix B: Transform Examples}

Let us demonstrate how to apply the above transform method to obtain the analytical expressions when $\Gamma$ are simple curves, which contain a small number of modes in their Fourier expansion.

\section{A. Elliptical border}

Let $\Gamma$ be an ellipse described by the equation

$$
\Gamma: \quad X^{2}+\frac{Y^{2}}{E^{2}}=a^{2}
$$

where $a$ is the minor ellipse axis size, and ellipticity $E \geq 1$. Observe that to provide the equivalence of $\mathrm{Eq}$. (B1) to the needed equation $u(X, Y)=u_{0}$ it is sufficient to keep only one mode in the expansion (A5), i.e. to put all the coefficients $C_{n}, \tilde{C}_{n}$ equal to zero except for $C_{1}, \tilde{C}_{1}(A 10)$

Equation $(B i)$ is rewritten as

$$
\left.\left(X_{1}^{2}+\frac{Y_{1}^{2}}{E^{2}}\right)\right|_{u=u_{0}}+\left.\cos 2 v\left(X_{1}^{2}-\frac{Y_{1}^{2}}{E^{2}}\right)\right|_{u=u_{0}}=2 a^{2},
$$

and we find

$$
\begin{aligned}
& \left.X_{1}\right|_{u=u_{0}}=a, \\
& \left.Y_{1}\right|_{u=u_{0}}=E a .
\end{aligned}
$$

Formula (B2) allows us to obtain the coefficients $C_{1}, \tilde{C}_{1}$ with certain degree of arbitrariness, because the quantity $u_{0}$ is not strongly determined.

Assuming $u \rightarrow+\infty$ at $X, Y \rightarrow \infty$, we obtain

$$
C_{1}=a \frac{E+1}{2} e^{-u_{0}}
$$




$$
\tilde{C}_{1}=-a \frac{E-1}{2} e^{u_{0}}
$$

If we choose

$$
u_{0}=\frac{1}{2} \ln \frac{E+1}{E-1}
$$

we get the known semi-elliptical coordinates

$$
\begin{aligned}
& X=a \sqrt{E^{2}-1} \sinh u \cos v, \\
& Y=a \sqrt{E^{2}-1} \cosh u \cos \cos v .
\end{aligned}
$$

These coordinates are known to separate variables for the Laplacian operator that is used for the solution of the elliptical plasma equilibrium problem. However, they cannot be applied to the situation when $E \rightarrow 1$.

Having the arbitrariness in the choice of $u_{0}$, we may put, for example, $u_{0}=0$. In this case our transform has a form

$$
\begin{aligned}
& X=a(E-1)\left(\sinh u+\frac{e^{u}}{E-1}\right) \cos v, \\
& Y=a(E-1)\left(\cosh u+\frac{e^{u}}{E-1}\right) \sin v,
\end{aligned}
$$

which results in usual polar coordinates at the limit $E \rightarrow 1 \quad(u \rightarrow \ln R)$.

The Jacobian of the considered transform

$$
J=\frac{1}{2}\left(X_{1}^{2}(u)+Y_{1}^{2}(u)+\cos 2 v\left(Y_{1}^{2}(u)-X_{1}^{2}(u)\right)\right)
$$

can be equal to zero only when $X_{1}=Y_{1}=0$, which is prohibited outside the ellipse $\Gamma$ due to $(B: 3)$.

Thus, the above transform (in particular, (B4) and (B5)) satisfies all three requirements mentioned in Appendix A.

\section{B. Moon-shaped border}

Let $\Gamma$ be described by the equation

$$
\left(X^{\prime}-\epsilon\left(X^{2}-Y^{2}\right)\right)^{2}+(Y+2 \epsilon X Y)^{2}-1+\epsilon^{2}\left(\left(1-2 \epsilon^{2}\right)\left(X^{2}+Y^{2}-1\right)+\epsilon^{4}-5 \epsilon^{2}+4\right)=0 .
$$


For an arbitrary $\epsilon<0.5$ this curve is similar to a moon-like deformed circle, which tends to be a usual unit circle at $\epsilon \rightarrow 0$. Substituting the general form (A5) of our transform into (B6) together with the condition (A12), and forcing all coefficients in front of the non-zero harmonics to equal zero, we find that the transform is

$$
\begin{aligned}
& X=e^{u-u_{0}} \cos v+e^{2\left(u_{0}-u\right)} \cos 2 v, \\
& Y=e^{u-u_{0}} \sin v-e^{2\left(u_{0}-u\right)} \sin 2 v .
\end{aligned}
$$

This describes the curve $\Gamma$ given by $(\mathrm{B} 6)$ at $u=u_{0}$ (the quantity $u_{0}$ can be chosen arbitrarily).

Let us prove the positivity of the Jacobian, which could be equal to zero only when

$$
\partial_{v} X=\partial_{v} Y=0
$$

(see Appendix A). L'sing the explicit expressions (B6), we find that the Jacobian equals zero for $u \geq u_{0}: e^{3\left(u-u_{0}\right)}=2 \epsilon$. That is impossible for $\epsilon<0.5$, which corresponds to the general condition (A1:3).

\section{Remark}

The analytical solution of the above-mentioned Dirichlet's problem can be found in the furm of an expansion wer different eigenfunctions. In particular, the spherical functions, the minlipole tharges and other functions can be used as a basis for such an expansion. However, the proposed mothod of the implicit Fourier expansion given in Appendix $A$ is often more andratl and complart in cases when the contour $\Gamma$ is a simple curve which contains a finite number of terms in a series (A.5). It should be noted that most of the boundary contours appearing in practical problems of electrostatics, or plasma equilibrium, or hydrodynamics rin be usually represented by such a finite sum (precisely or approximately), while the representation by means of another basis usually needs many terms of an infinite series to whain comparable accuracy. 


\section{Acknowledgments}

The authors are grateful to A.Yu. Sokolov and A.A. Subbotin for useful discussions and assistance in the development of numerical codes. This work was supported by U.S. Department of Energy contract \#DE-FG05-80ET-53088.

\section{DISCLAIMER}

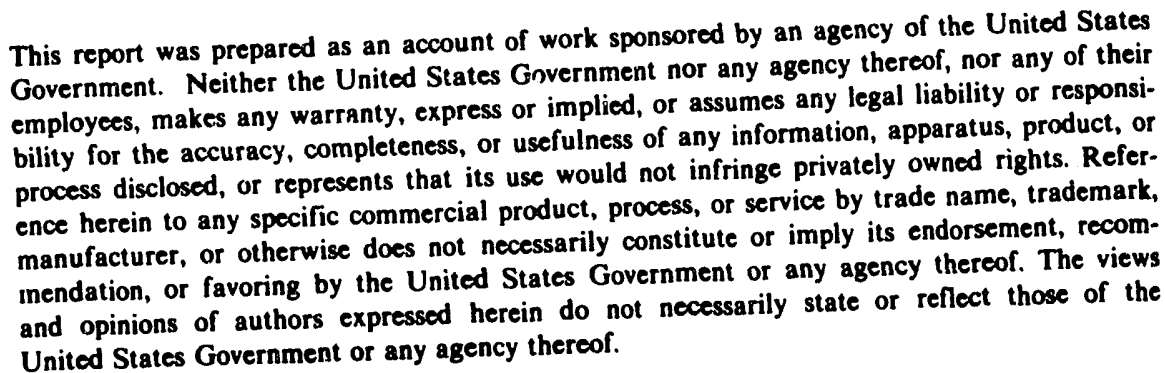




\section{References}

${ }^{1}$ J.G. Cordey, C.J.H. Watson, Proc. $5^{\text {th }}$ European Conf. on Controlled Fusion and PLasma Physics (Grenoble, 1972), Vol. 1, p. 98.

${ }^{2}$ J.G. Cordey, C.J.H. Watson, Fusion Reactor Design Problems, Nucl. Fusion Suppl., Vienna, p. 199 (1974).

${ }^{3}$ V.P. Pastuhov, H.L. Berk, accepted for publication in Nucl. Fusion (1993).

${ }^{4}$ F.N. Coensgen, T.A. Casper, D.I. Correll, C.C. Damm, A.H. Futch, B.G. Logan, A.W. Molvik, and R.H. Bulmer, UCRL 9i280, Rev. 1, LLNL (1987).

${ }^{5}$ I.A. Kotelnikov, V.V. Mirnov, V.P. Nagornyi, and D.D. Ryutov, Proc. $10^{\text {th }}$ IAEA Conf. on Plasma Phys. and Controlled Nucl. Fusion Res. (Vienna, 1986) Vol. 2, p. 309.

${ }^{6}$ F.A. Coensgen, J.F. Clauser, D.L. Correll, IV.F. Cummins, C. Gormezano, B.G. Logan. A.II. Molvik. II.E. Nexsen, T.C. Simonen. B.II. Stallard, and W.C. Turner, Proc. $6^{\text {th }}$ l.1E.d Conf. on Plasma Phys. and Controlled Nucl. Fusion Res. (Vienna, 197i) Vol. 3, p. 135

'L.C. Steinhauer. Physs. Fluids B 2, 3081 (1990).

${ }^{s_{H}}$ H. Wi, M.E. Oakes, Phys. Fluids B 3, 2113 (1991).

${ }^{9}$ L.D. Pearlstein, T.B. Kaiser, and W.A. Newcomb, Phys. Fluids 24, 1326 (1981).

${ }^{10}$ D.A. D'Ippolito and J.P. Freidberg, Phys. Fluids 25, 1617 (1982).

"S.P. Ilirshman, IV.I. Van Rij, and P. Merkel, Comput. Phys. Commun. 43, 143 (1986). 
${ }^{12}$ K. Watanabe, N. Nakajima, M. Okamoto, Y. Nakamura, M. Wakatani. Nucl. Fusion 32, 1499 (1992).

${ }^{13}$ G.V. Stupakov, Sov. J. Plasma Phys. 5, 486 (1979).

${ }^{14}$ L.E. Zakharov, V.D. Shafranov, in Reviews of Plasma Physics Vol. 11, edited by B.B. Kadomtsev (Consultants Bureau, New York, 1985). 


\section{Figure Captions}

1. Structure of magnetic field lines in the toroidally linked mirror system.

2. Typical constant pressure contours at the middle of toroidal cells with $\tilde{E}=4$
(a) $\lambda=0$; (b) $\lambda=0.2$; (c) $\lambda=0.8$;
1) plasma boundary, 2) plasma column center.

3. Typical constant pressure contours at the middle of toroidal cells with $\tilde{E}=13$
(a) $\lambda=0.2$; (b) $\lambda=0.8$
1) plasma boundary, 2) plasma column center.

4. Plasma boundary position

1) $\tilde{E}=13,2) \tilde{E}=4$. 


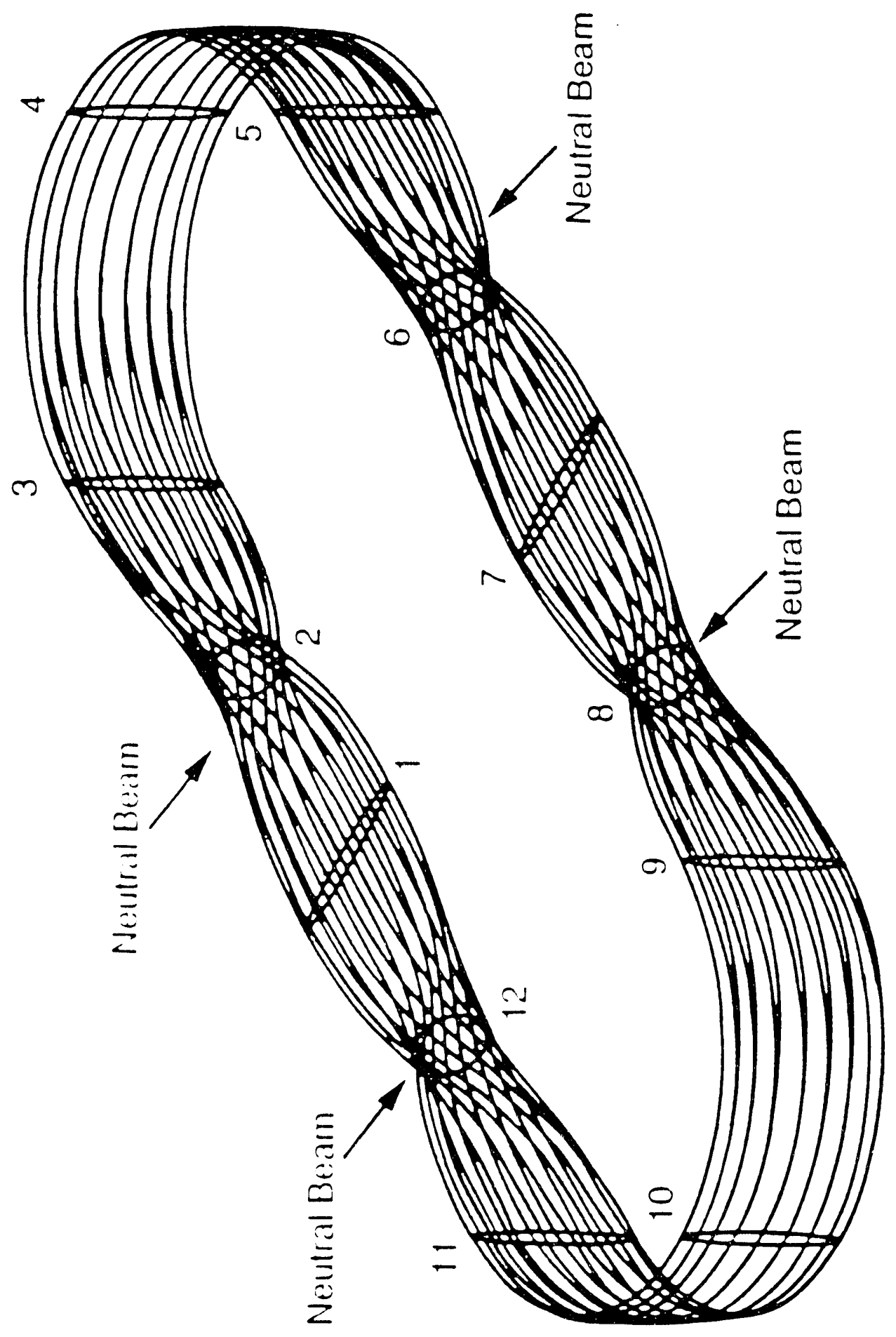




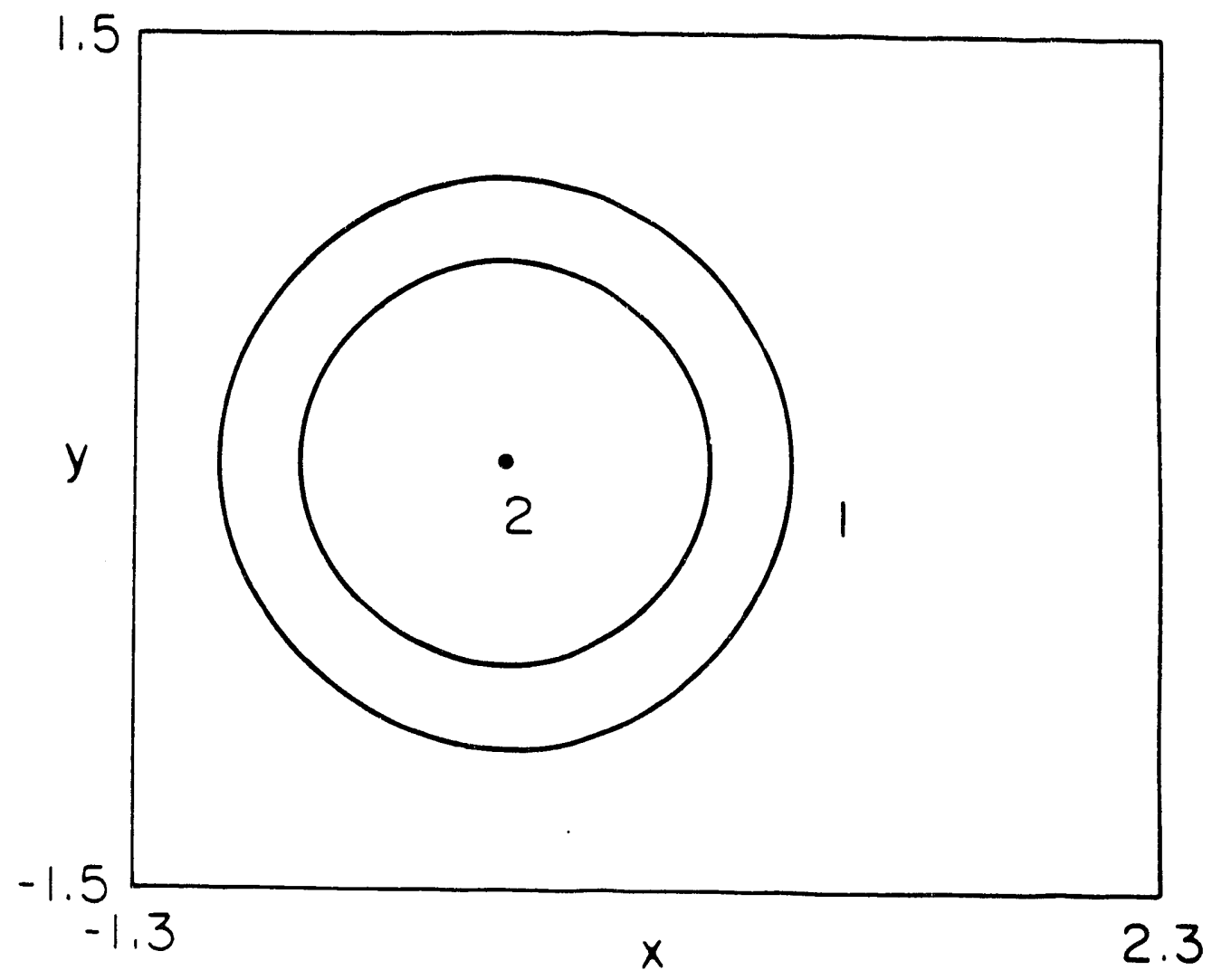

Fig. $2 a$ 


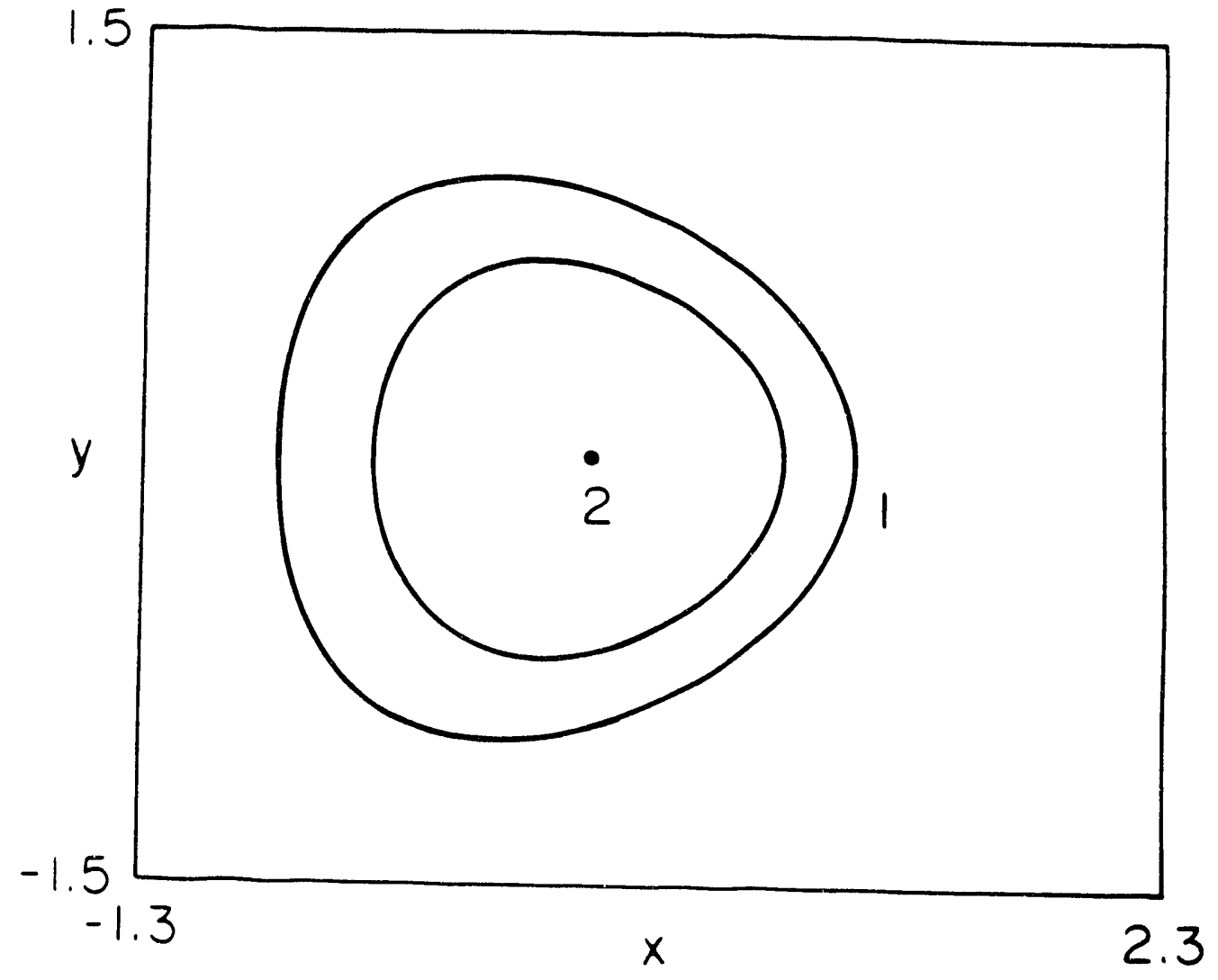

Fig. Ib 


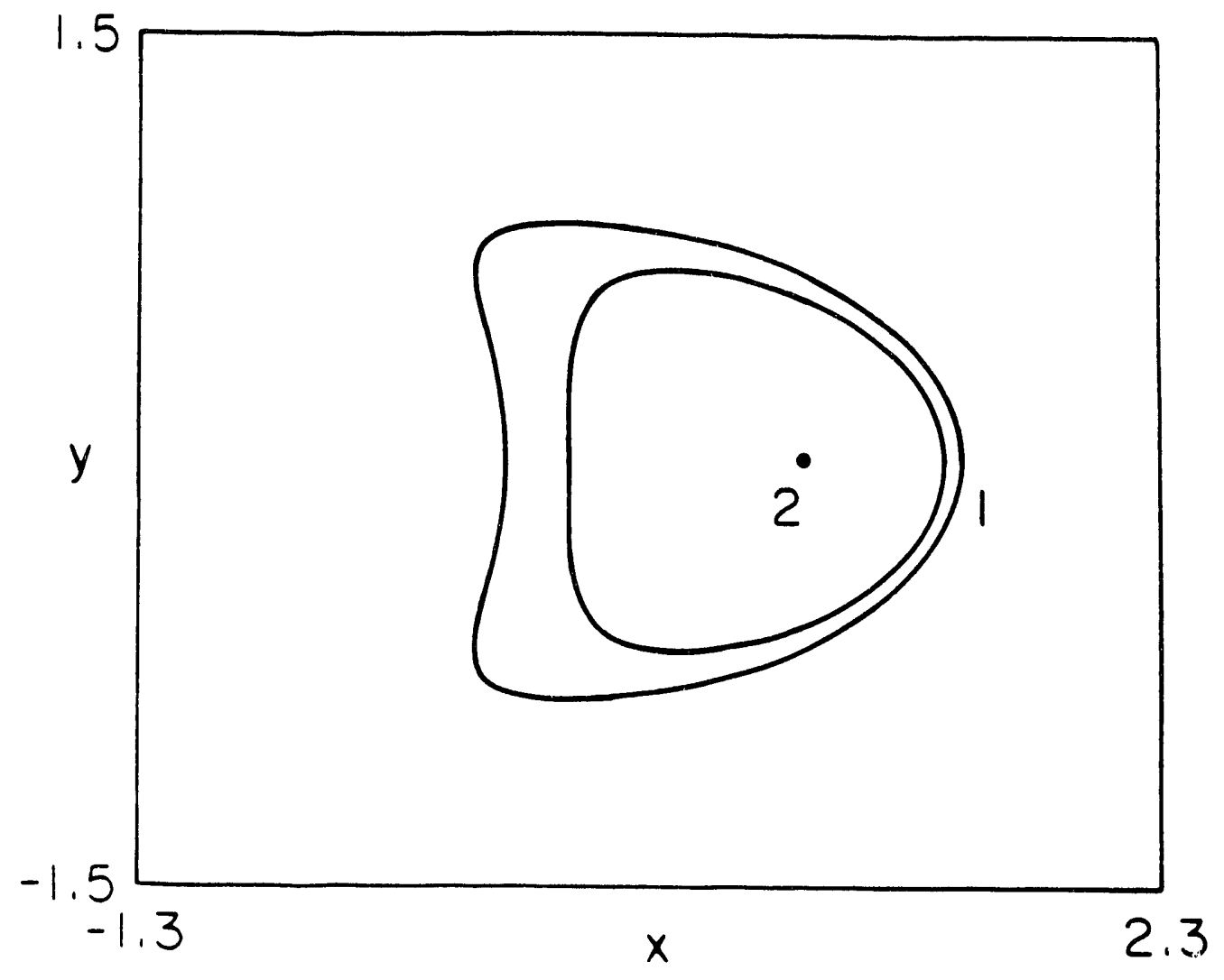

Fig. 2C 


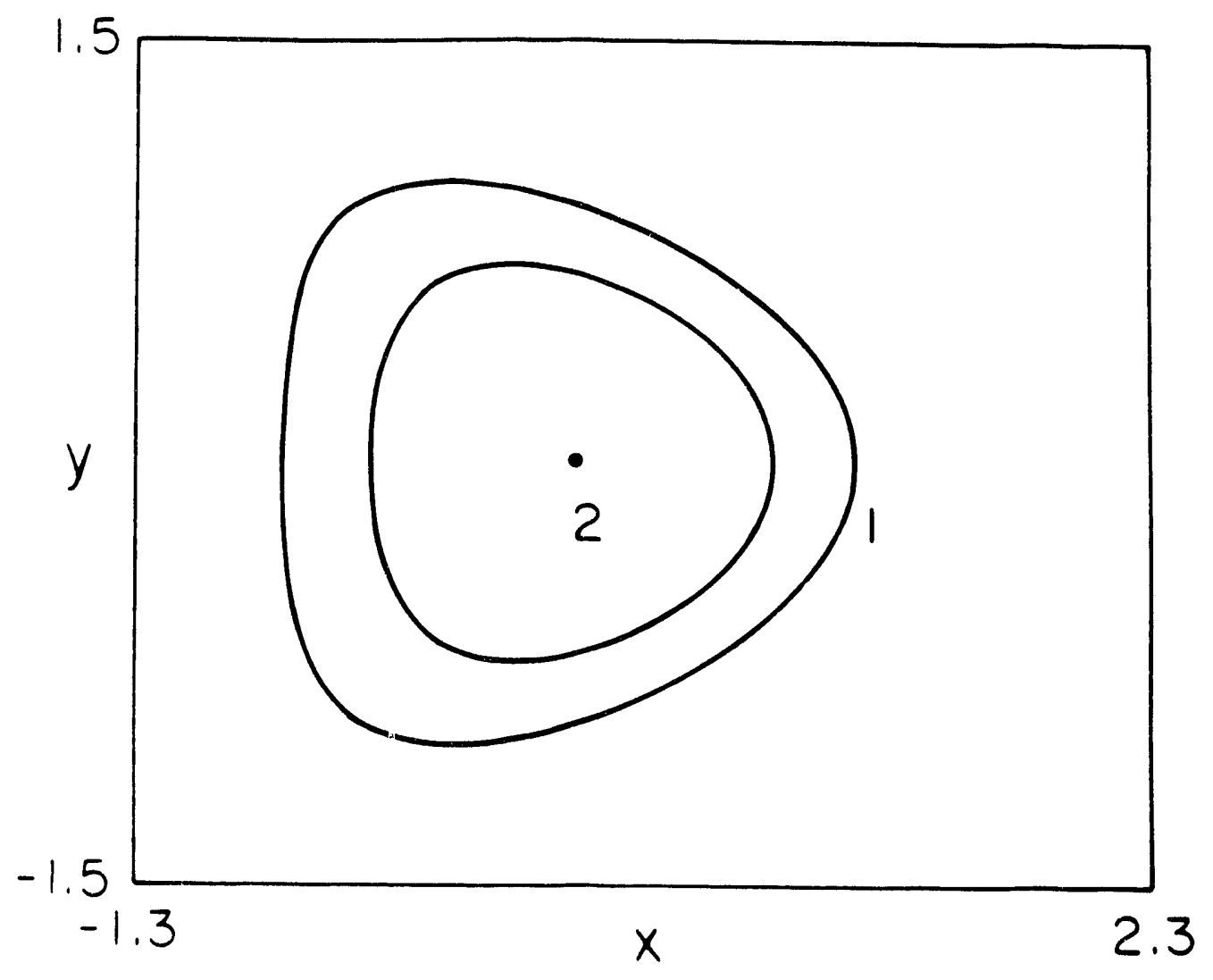

Fig. ja 


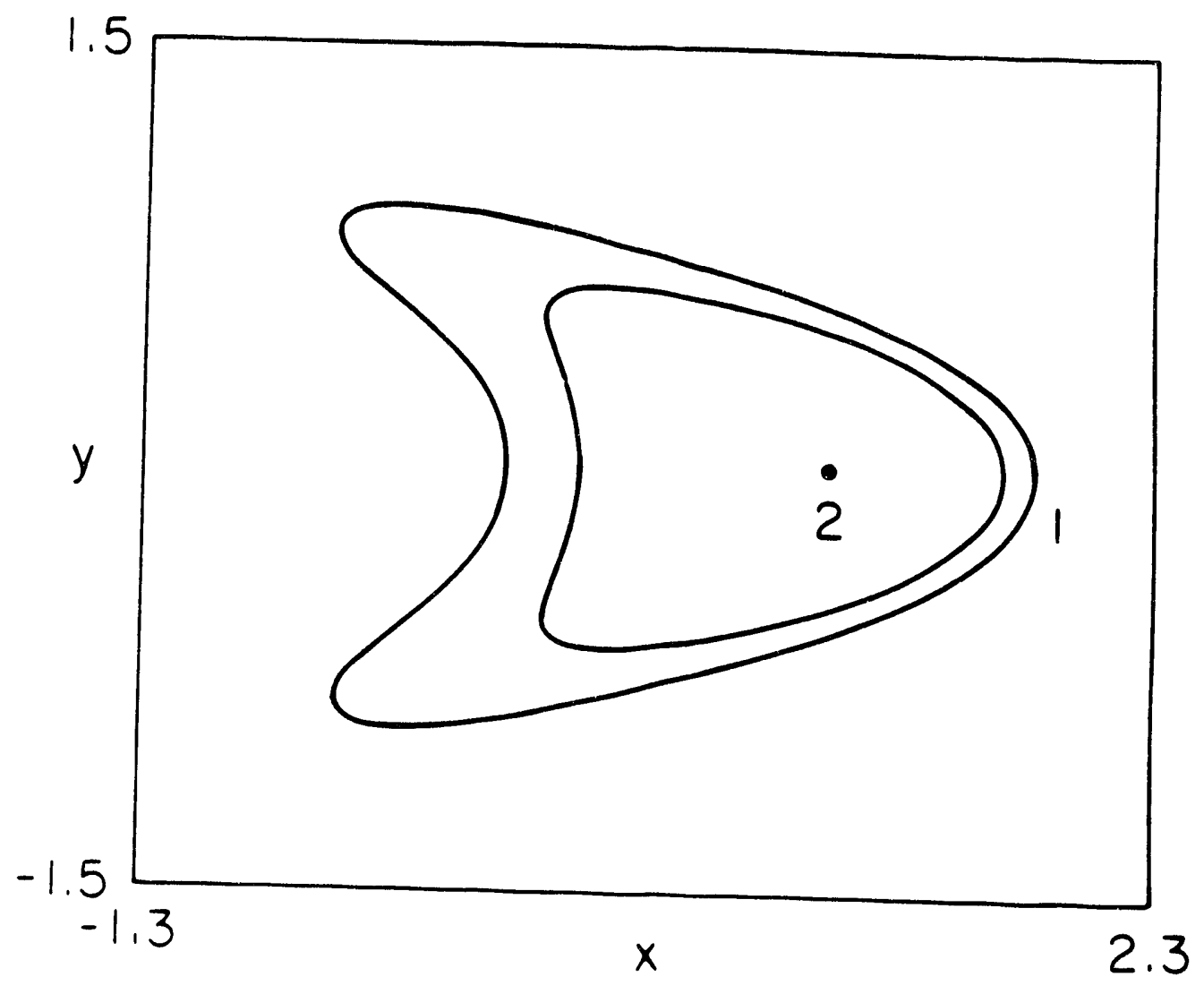

cig. 3b 


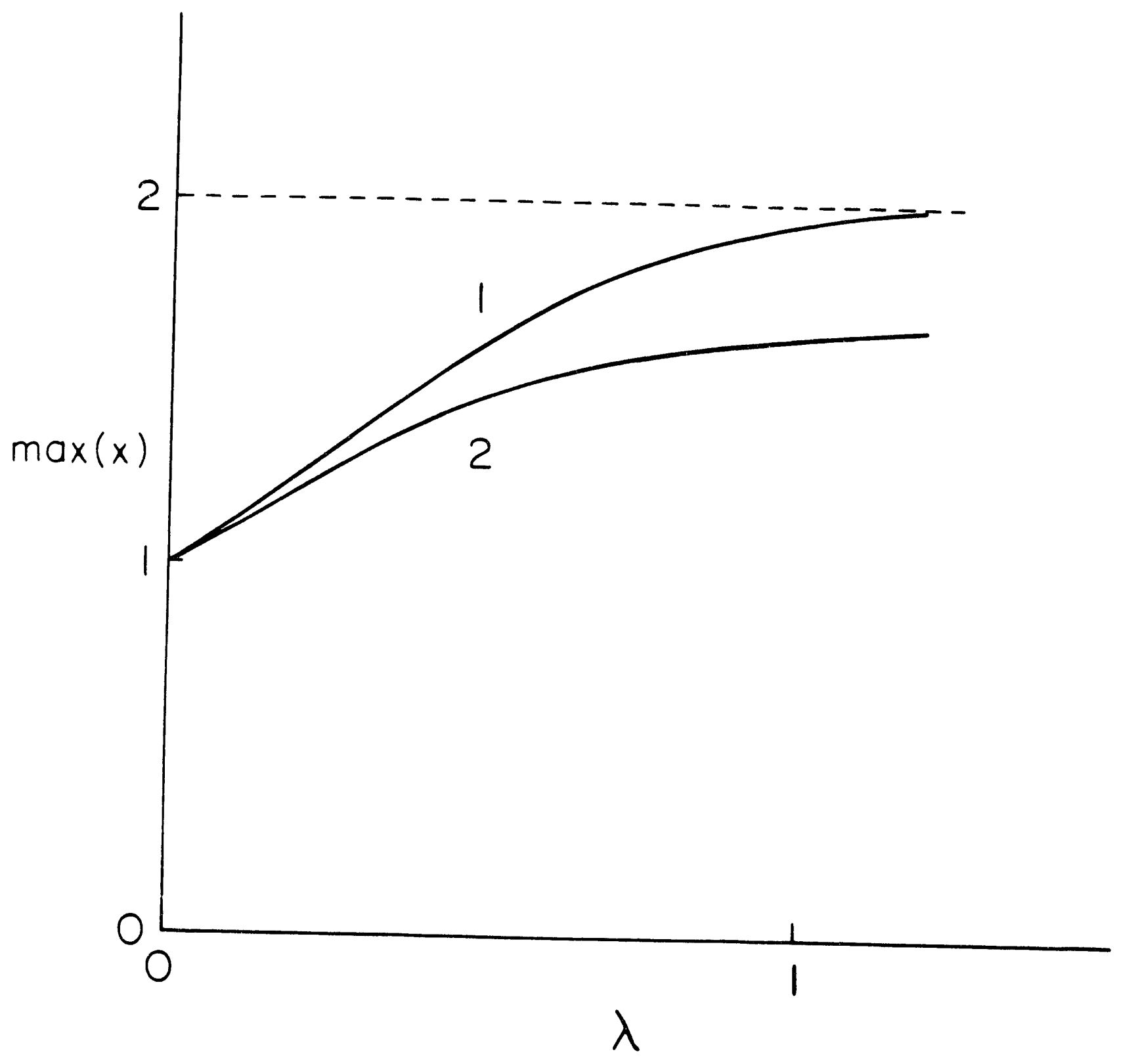

Fig. 4 

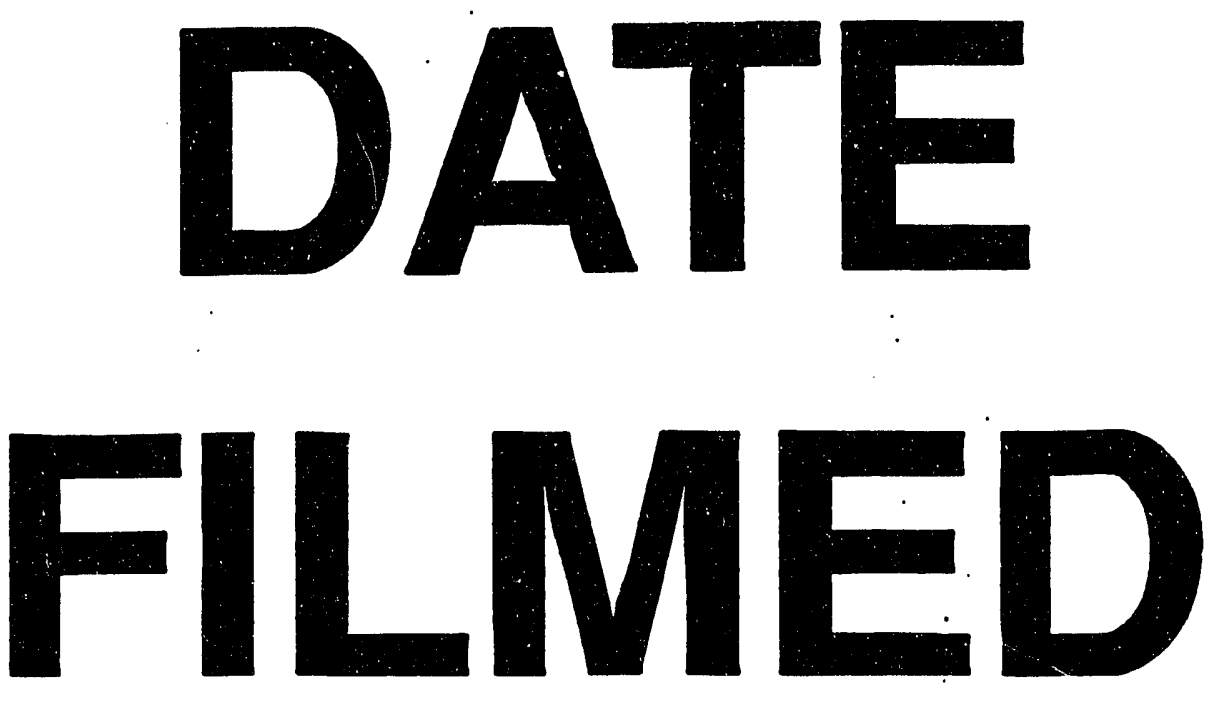

$10 / 12 / 93$
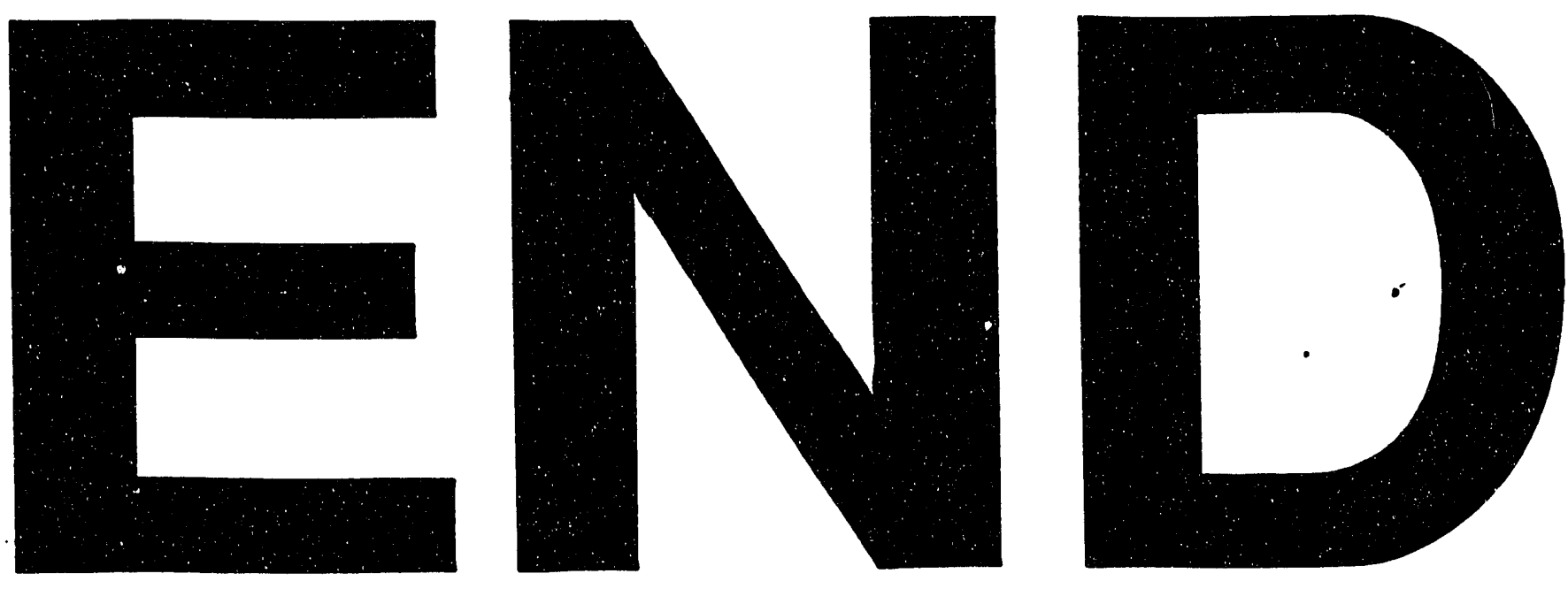
